\title{
ERRATUM 1
}

Article "Feelings of pleasure of nurses working in primary care", with number of DOI: http://dx.doi.org/10.1590/0034-7167-2018-0518, published in the journal Revista Brasileira de Enfermagem, v72(4): 1036-43, on page 1036:

Where it read:

\section{Sandra Maria de Andrade"}

Rocha GSA, Andrade SM, Silva DMR, Terra MG, Medeiros SEG, Aquino JM. Feelings of pleasure of nurses working in primary care. Rev Bras Enferm. 2019;72(4):1036-43. doi: http://dx.doi.org/10.1590/0034-71672018-0518

Read:

\section{Maria Sandra de Andrade"}

Rocha GSA, Andrade MS, Silva DMR, Terra MG, Medeiros SEG, Aquino JM. Feelings of pleasure of nurses working in primary care. Rev Bras Enferm. 2019;72(4):1036-43. doi: http://dx.doi.org/10.1590/0034-71672018-0518 